\title{
Resilient Self-Debugging Software Protection
}

\author{
Bert Abrath, Bart Coppens, Ilja Nevolin, and Bjorn De Sutter \\ Computer Systems Lab, Ghent University, Belgium \\ bert.abrath@ugent.be,bart.coppens@ugent.be,ilja@nevolin.be,bjorn.desutter@ugent.be
}

\begin{abstract}
Debuggers are a popular reverse engineering and tampering tool. Self-debugging is an effective technique for applications to defend themselves against hostile debuggers. In penetration tests on state-of-the-art self-debugging, we observed several opportunities through which it could be attacked, however. We therefore improved upon the existing technique, making it more resilient by introducing reciprocal debugging and making the transfers of control between protected application and self-debugger more stealthy. This paper presents the improved self-debugging design, and details our research efforts into realizing reciprocal debugging. In our evaluation we show that the improved design is significantly harder for attackers to defeat.
\end{abstract}

Index Terms-reverse engineering, software protection, antitampering, anti-debugging, self-debugging

\section{Introduction}

Debuggers are a popular tool in the toolbox of socalled man-at-the-end (MATE) attackers [1]. These attackers try to gain unauthorized access to confidential assets in software by reverse engineering the software and try to tamper with the integrity of software assets by manipulating them, e.g., to work around license checks or copy protections. Debuggers offer a convenient, interactive, scriptable, and flexible interface to inspect and tamper with the evolving internal state of a running program.

Those same attacker goals can be reached with many other means available to MATE attackers that have whitebox access to the software and the systems on which they attack it in their labs. However, alternative tools such as emulators, instrumentation tools, and hypervisors lack the convenience of debuggers for many concrete actions that attackers want to perform. Preventing the use of debuggers can hence slow down MATE attackers, and force them to invest more effort and hence more money into their attacks. As the major objective of software protection is to change a MATE attack's (perceived) balance between required attack investment and resulting profit [2], [3], techniques that prevent the use of hostile debuggers can hence be a worthwhile defensive investments.

One class of anti-debugging techniques has the application query the environment (e.g., via standard library APIs or system calls) for signs they are being debugged

NB: appendices, if any, did not benefit from peer review.

A preprint of this paper has been deposited on ArXiv.

This research was funded by the Fund for Scientific Research - Flanders (FWO) as part of project grant 3G0E2318; by the Flemish Government's Cybersecurity Initiative Flanders; and by the TETRAMAX project, which has received funding from the European Union's Horizon 2020 research and innovation programme under grant agreement number 761349. or not [1], [4], [5]. However, as debuggers can intercept those queries, they are easy to work around. For popular debuggers such as OllyDbg, a range of plugins exist to defeat such simple anti-debugging techniques [6]-[8].

A stronger form of anti-debugging is by means of self-debugging [9], [10]. The application then contains its own so-called self-debugger and spawns its own debugging process, which attaches itself to the application as a debugger soon as it is launched. As all major OSs only support one debugger process per debuggee [11]-[13], the application-friendly self-debugger occupies the only seat available, such that a hostile debugger can no longer be attached. The self-debugger is application-friendly, in the sense that it does not contain the traditional debugger functionality that can be used to attack the application.

To prevent an attacker detaching the self-debugger to free the seat for their own hostile debugger, we previously proposed to migrate critical functionality from the application to the self-debugger [14]. Through source code annotations, a developer can designate the code to be migrated. During execution, at certain program points control is transferred from the protected application to the self-debugger. The latter subsequently executes the migrated (and transformed) fragments, and passes control back to the application. Simply detaching the selfdebugger then breaks the program. Consequently, attacking a self-debugging program to re-enable the use of a debugger requires much more work from the attacker, such as migrating the necessary functionality back into the application (or into the dynamically linked library, which we can protect as well). The usefulness of such antidebugging is apparent from the fact that our approach is deployed in millions of devices protected by Nagravision, the company to which we transferred our technology.

Still, attackers have some opportunities to attack the self-debugging protection. In a public challenge and in professional penetration testing experiments [15], we observed two critical aspects. First and foremost, if an attacker can still debug the self-debugger, they can observe the migrated functionality and the control transfers. This can help them to work around the protection by undoing it or by circumventing it in some way. Secondly, when the code fragments implementing the transitions and the interface to pass data between the application and the self-debugger lack stealth, attackers can use relatively simple static techniques such as pattern matching to identify them, easing the implementation of (semi-)automated workarounds. We observed both attacks in the aforementioned penetration testing experiments.

To counter those activities, we studied extensions in two directions: more stealthy control flow transfers that are also more resilient to workarounds on the one hand, 
and reciprocal debugging on the other hand, because when both the protected application and the self-debugger debug each other, an attacker can debug neither of them.

This paper reports our findings. Both for making control transfers more stealthy and resilient, and for making self-debugging reciprocal, we report opportunities and obstacles as present on popular architectures and operating systems. We make the following contributions:

- We present an improved design of our selfdebugging technique, featuring reciprocal debugging and stealthier interfaces.

- We describe a working reciprocal debugging implementation, and explain the deadlocks inherent in such a system and how to handle them.

- We examine portable methods through which a debugger can be stealthily signaled.

- We provide a security evaluation of our improved design, using Binary Ninja.

- We provide an open-source prototype implementation of our design. Our framework is available at https://github.com/aspire-fp7/framework, with the code related to self-debugging available separately at https://github.com/csl-ugent/anti-debugging.

\section{Self-Debugging Security Analysis}

MATE attackers want to analyze or tamper with the sensitive assets contained in a target application, and can be assumed to have total control over the environment this application runs in [16]. This means they can run the application in an emulated environment, disable security features provided by the OS such as ASLR, as well as use many other different tools at their disposal to analyze or tamper with an application statically or dynamically. Debuggers are powerful dynamic tools, providing attackers with the capabilities to gather traces, dump cryptographic keys, or even execute code fragments out of context, e.g., to work around copy protections.

All major OSs only provide support for a single debugger per application [11]-[13]. Self-debugging takes advantage of this limitation by occupying the only available debugger seat with a custom, application-friendly selfdebugger [14]. This protection prevents attackers from attaching their own, hostile debugger to the application, and consequently first has to be defeated by any attackers before they can debug the application. The protection has thus become the target, and we therefore investigate how it can be attacked as well as defended.

We start with an overview of our original design, and then explore the different ways in which it can be attacked.

\subsection{Self-Debugging Overview}

Figure 1 illustrates the design of the original selfdebugging technique [14]. Whereas the original, unprotected application as shown on the left consists of a single process, the protected self-debugging application, which was generated by means of binary rewriting, consists of two processes. The first process is the protected application, which still contains the main part of the application code, largely unchanged. The second process is the selfdebugger, which provides the protection to the application by "debugging" it. In the context of this paper, this means "attaching to the application like a debugger does", it does not include the act of using debugger functionality to find bugs. The self-debugger contains a mini-debugger component that implements the necessary debugger interface functionality using ptrace, the standard debugging API on Linux [11]. Next to that it also contains a number of code fragments migrated from the application context, guided by source code annotations. These fragments get invoked by the protected application whenever they need to be executed, but they will be executed in the selfdebugger's context. It is this mechanism that creates a dependence between the protected application and the self-debugger. This dependence prevents the execution of the application when the self-debugger is not attached, and thus keeps an attacker from detaching the self-debugger and attaching their own hostile debugger instead.

The initialization of the self-debugging technique is provided by an initialization routine that is injected into to the binary. This routine is invoked whenever the binary is loaded, both in case the binary is an executable file with which a process is launched and in case it is a dynamically linked library loaded into an existing process. The routine performs a fork [17], with the parent becoming - or rather, staying - the protected application, and the child becoming the self-debugger that attaches to the application. The application thread that invokes the initialization routine is kept waiting and only allowed to continue after the self-debugger has finished attaching to all of the protected application's threads. There is also an accompanying finalization routine.

At run time, the protected application needs to execute migrated code fragments. The red edges in Figure 1 show the flow of control to invoke and execute such a fragment in the self-debugger. The application contains exceptioninducing instructions at every location a fragment is to be invoked. In our original implementation, these exceptioninducing instructions are hard-coded breakpoint (BKPT) instructions preceded by instructions that push an identifier onto the stack. When they get executed an exception occurs that generates a signal. The kernel then stops the protected application until its debugger investigates the signal and allows the application to continue. The exception thus switches control flow to the self-debugger, and more specifically to its mini-debugger component, which derives the requested code fragment from the identifier on the stack: It uses this identifier to look up the associated fragment's address in a mapping table, and then transfers control to that address. The migrated fragment subsequently executes in the self-debugger context, but performs reads and writes to the protected application's address space when required, using the mini-debugger's memory support. Upon return from the fragment, the mini-debugger updates changed registers in the application process, and finally allows it to continue executing. The mini-debugger itself then continues in its debugger loop, awaiting further requests by the application.

\subsection{Defeating Self-Debugging}

To attach their own hostile debugger functionality to the protected application to observe or manipulate its execution, attackers first have to defeat the self-debugger. 


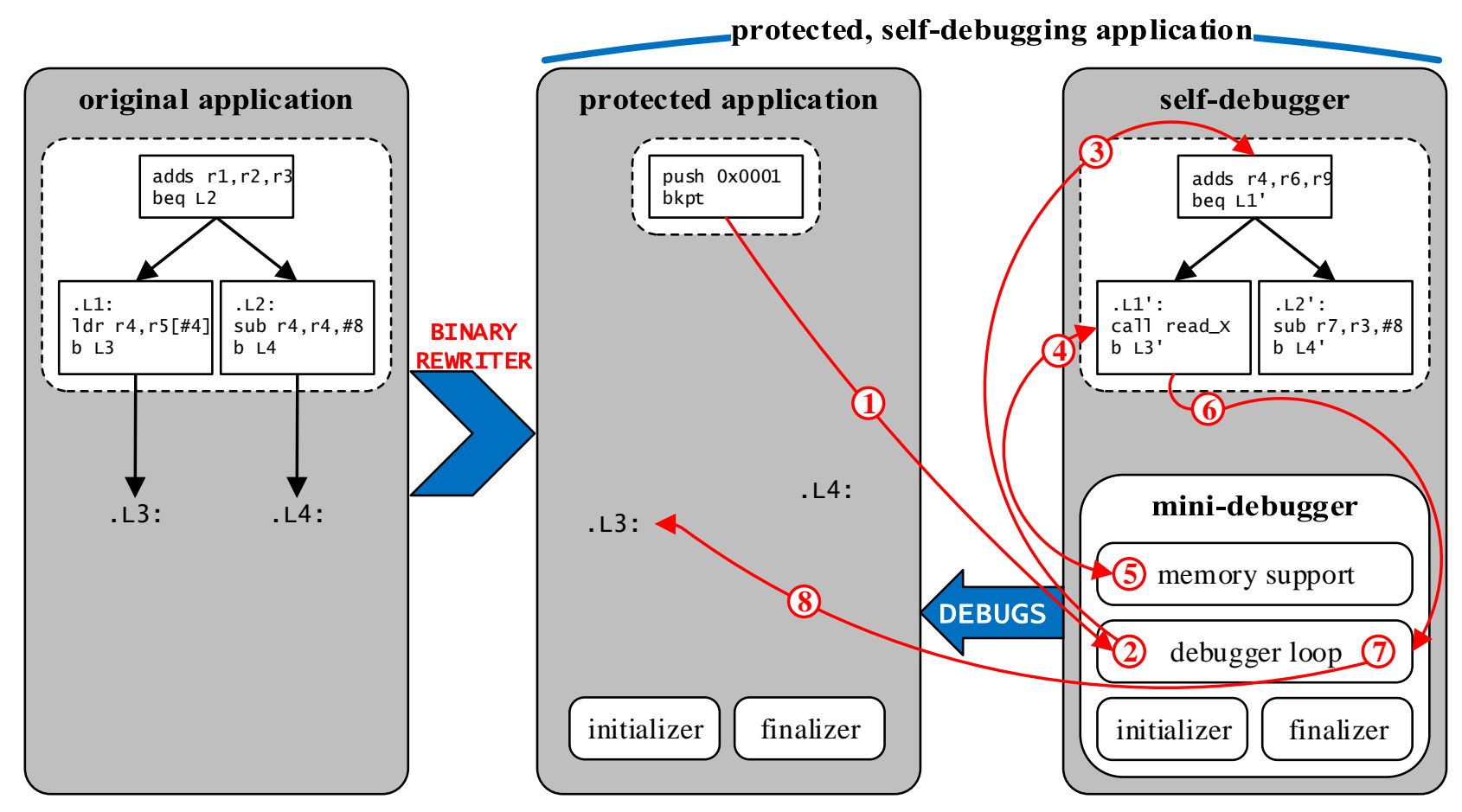

Figure 1. Overview of the original self-debugging protection

They can achieve this in a number of ways. We present these attacks in two broad categories: those where the selfdebugger is detached, and those where it is still attached. In the first category a hostile debugger can be attached directly to the protected application. In the second category, the hostile debugger cannot be attached directly.

2.2.1. With the Self-Debugger Detached. Detaching the self-debugger is not hard by itself. It can be done, e.g., by killing the self-debugger, or by removing or disabling the initialization routine that spawns it. This by itself does not suffice, however, because the protection also includes the migration of code fragments into the self-debugger. As the protected application at times needs to execute these code fragments, it will stop working when the selfdebugger is detached. Attackers thus need to deal with these migrated code fragments. They can do so in several ways: The migration transformations can be reversed, the execution of fragments can be avoided, and they can be emulated in an attacker-controlled process.

Reversing the transformations means both undoing the changes to the migrated code fragments so they can execute directly in the application again, and reversing the control flow switches to the mini-debugger back to normal control flow that does not depend on a debugger. To reverse transformations in general, attackers first need to find out which transformations have been applied and where, after which they can tamper with the binary to undo the protection. For self-debugging, attackers need to locate the control flow switches and the migrated code fragments. Simple static analysis suffices to do so: one can directly find the hard-coded breakpoints by simply scanning the binary, and one can find their intended targets by using the accompanying identifier to look up the target address in the mapping table. Alternatively, dynamic analysis such as tracing the self-debugger's execution can also be used to determine the intended targets of the control flow switches. Such an analysis can be achieved easily by attaching a hostile debugger to the self-debugger process. In the public challenge of the ASPIRE FP7 project, the self-debugging protection was reversed manually by an amateur hacker in this way [15], [16]. With the necessary effort, attackers could automate these analysis steps, as well as the subsequent tampering.

Alternatively, attackers might be interested in tracing or tampering with only a small part of the application, in which case reversing all the transformations for all of the migrated code fragments is typically not necessary. The attackers can instead try to invoke execution paths that enable them to reach their specific goals, yet contain no (or few) migrated code fragments. Using a debugger to alter the flow of execution at will-perhaps even in a scripted manner-is of great benefit in constructing such paths. Moreover, when the code to be protected is a dynamically linked library, attackers can invoke exposed API functions in the order of their choosing. This attack approach was employed by professional penetration testers during the ASPIRE FP7 project's security evaluation [15], [16]. The same analyses as used for reversing transformations are required, as well as the extra path analysis.

A last attack option is to leave all transformations intact, and to emulate the mini-debugger function. This emulation comes down to switching control flow to the right migrated code fragments when requested, and creating the right context for them to execute. One way to do so is to create and attach a hostile debugger that contains the necessary emulation functionality next to the traditional debugging functionality one expects to find in any typical debugger. The migrated code fragments can then be executed in this hostile debugger's context, while it can use its privileges to tamper with and trace the protected application at will. Another option would be to 


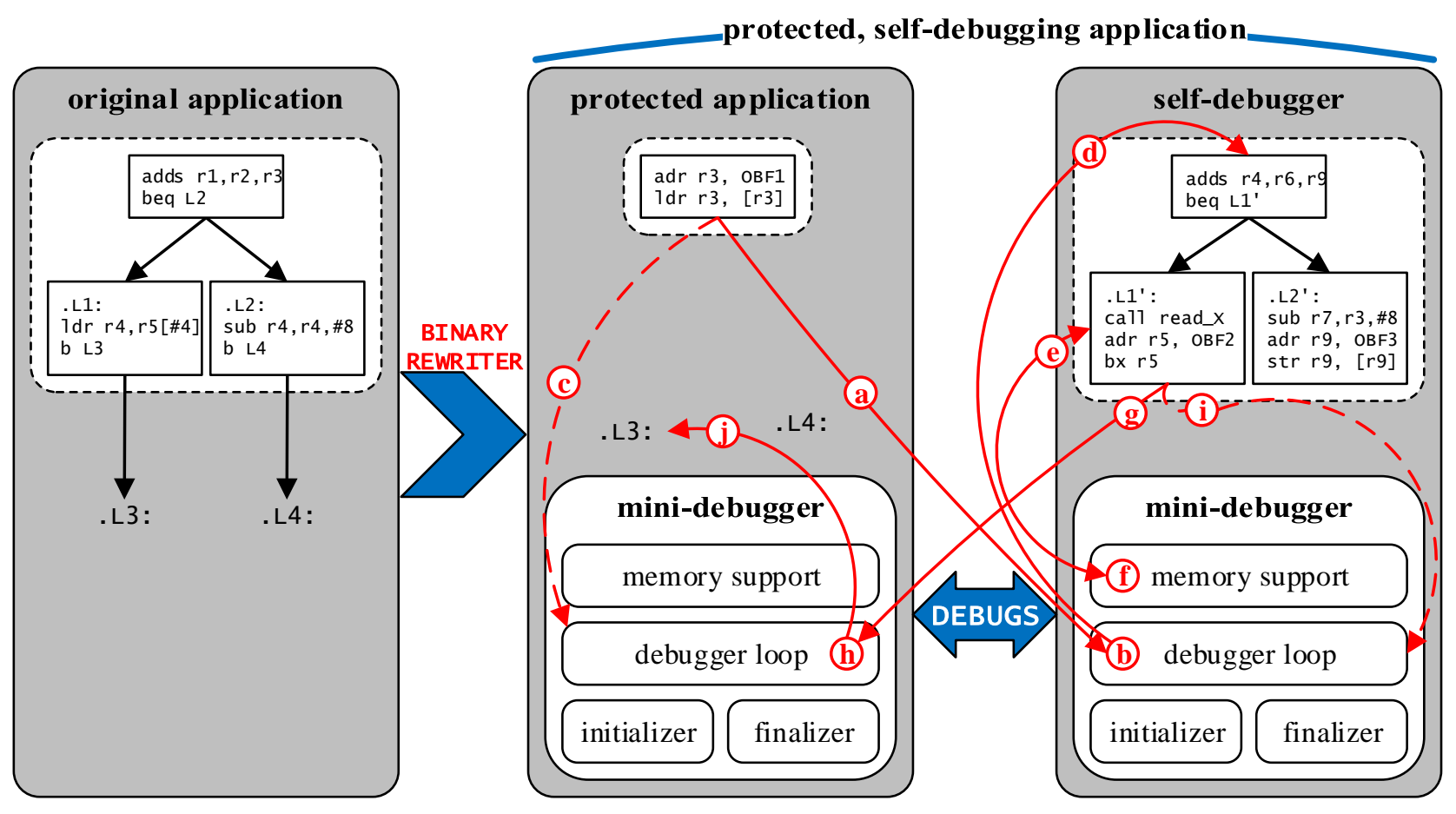

Figure 2. Overview of the improved self-debugging protection featuring reciprocal debugging and stealthier interfaces

insert some kind of signal handler that can resolve the control flow switches into the application itself. In that scenario, however, the migrated code fragments would run directly in the target's address space. As the fragments were transformed to run in another address space, some hooking of the ptrace API would be required for these fragments to execute correctly.

2.2.2. With the Self-Debugger Attached. Here, attackers cannot attach their own debugger to observe or to tamper directly with the protected application. However, they can target the self-debugger itself, or gain debugging privileges through some other way than the ptrace API. During the ASPIRE Security Evaluation professional penetration testers tried and failed to use multiple tools for tracing or live-debugging a protected application [15].

Attackers can attach their debugger directly to the selfdebugger, and use its privileges to subvert the protection, effectively turning the self-debugger into their own hostile debugger. By debugging the self-debugger they have direct access to the migrated code fragments during their execution. More importantly, they can also gain indirect access to the protected application by taking advantage of the self-debugger's privileges and injecting ptrace requests. This allows them to trace and tamper with the application, without directly debugging it or interfering in its workings. Such an approach would require little analysis, yet bypass the protection completely.

Alternatively, debugging-like powers can also be achieved without using the ptrace API. Program emulation using Valgrind [18], or QEMU [19] can provide tracing, as long as the ptrace API and the entire workings of the control flow switches are correctly emulated. Using Valgrind, interactive debugging can even be provided through a gdbserver. We tried attacking our original implementation using this approach, but did not succeed because of incorrect emulation of the ptrace API and the exception-inducing instructions [14]. Implementing a correct emulation of these aspects is just engineering, however. We therefore consider it worthwhile to defend the application against being executed in an emulated environment. Many such defenses already exist, and are orthogonal to our technique [20]. We thus consider antiemulation out of scope for this paper.

Finally, attackers could modify the OS in order to provide them an alternative to the ptrace API. This would come at the cost of considerable engineering effort, however. To be useful to attackers, such an API has to provide the functionality necessary for interactive debugging or performing scripted attacks.

\section{Resilient Self-Debugging Design}

To counter the above attacks, our improved selfdebugging technique features two improvements: a tighter, reciprocal coupling between the application process and its self-debugger process, and stealthier interfaces for invoking the mini-debugger. Figure 2 contains an overview of the design. It depicts both reciprocal debugging (i.e., both processes debugging each other) and stealthier interfaces (i.e., no more BKPT instructions). We discuss these aspects in detail below.

\subsection{Reciprocal Coupling}

The self-debugger can be a target for attackers, who either want to debug it or run the protected application without it being attached. We therefore explored methods of making the coupling between the two tighter, that is, making it harder for an attacker to kill, detach, or subvert the self-debugger without triggering a reaction. More specifically, we explored reciprocal debugging, where not 
only does the self-debugger "debug" the application, but the application itself also "debugs" the self-debugger. This keeps any hostile debuggers from attaching to either. Figure 2 presents an overview of reciprocal debugging, together with the changes to the original design it requires in terms of components and the flow of control. These changes serve to ensure that the protected application remains attached to the self-debugger. In the original design, the protected application could raise exceptions to invoke the mini-debugger. In the improved design, such invocations can happen in two directions. Consequently, both processes contain an identical mini-debugger.

With two mini-debuggers present and the accompanying bidirectional mini-debugger invocations, the interactions between the protected application and the selfdebugger are more complex. We start with an abstract overview of these interactions, and follow up by illustrating the control flow portrayed in Figure 2 as a concrete example. At any time during the execution of application code, either the protected application or the self-debugger is functioning as the exception-throwing process, with the other process functioning as the exception-catching process. The exception-throwing process executes (possibly migrated) application code, until it throws an exception to invoke the mini-debugger. The exception-catching process, on the other hand, executes in the debugger loop of its mini-debugger, awaiting any invocations. We consider the exception-catching process to contain an active minidebugger. When an exception is thrown by the exceptionthrowing process, this process is stopped, and the active mini-debugger in the exception-catching process is invoked. At this point, the roles of the two processes have to switch. The exception-catching process will transfer control to application code and become the exceptionthrowing process. Conversely, the exception-throwing process will ready itself for invocations by activating its mini-debugger, and thus become the exception-catching process. These transitions are managed by the active minidebugger in the exception-catching process. It first uses its debugger privileges to intervene in the stopped exceptionthrowing process, transitioning it to an exception-catching process. More precisely, the active mini-debugger manipulates the state and context of the exception-throwing process and then resumes it in the debugger loop, thereby activating its mini-debugger. After this transition, the active, invoked mini-debugger can complete the role switch by transferring control to the requested application code, thereby deactivating itself and transitioning its process from exception-catching to exception-throwing.

We present the control flow depicted by the alphabetically ordered red edges in Figure 2) as a concrete example. Here, the protected application is the exceptionthrowing process, executing application code; the selfdebugger is the exception-catching process, with an active mini-debugger. The protected application throws an exception (a), which invokes the active mini-debugger running in the self-debugger (b)). First, the active mini-debugger transitions the protected application to an exceptioncatching process by activating its mini-debugger (). Secondly, the active mini-debugger transitions the selfdebugger to an exception-throwing process by transferring control to application code, in the form of a migrated code fragment (d). This migrated code might perform memory operations, requiring the memory support of the minidebugger (e) and $\oplus$ ). At some point the migrated code in the self-debugger throws an exception (g), invoking the active mini-debugger running in the protected application ((1)). This active mini-debugger also manages two transitions: First, of the self-debugger into an exceptioncatching process (i)); Second, of the protected application into an exception-throwing process, by transferring control to application code (ij).

As an additional protective measure, the protected application and self-debugger attach to each other with the PTRACE_O_EXITKILL option enabled. This option ensures that a debugged process can never escape its debugger's control, even if the debugger unexpectedly exits. The kernel enforces this by immediately shutting down the debuggee when a debugger exits. If the debugger simply detaches, however, the debuggee is allowed to continue normally. In the case of reciprocal debugging, enabling this option ensures an attacker can kill neither the protected application nor the self-debugger, without the other immediately shutting down.

If the entirety of its initialization happens correctly, this design is impervious to dynamic tampering through a debugger. Once the initialization is done, the protected application can no longer be debugged, the self-debugger cannot be debugged, and even killing the self-debugger will not help an attacker.

\subsection{Stealthier Interfaces}

Regardless of the specific attack, analyzing how the mini-debugger is invoked and to which (migrated) application code it transfers control helps the attacker in understanding the application. In our original design, the interfaces between the protected application and minidebugger were implemented with manifest idioms (i.e., BKP T instructions and IDs pushed onto the stack), and the control flow between them was hence easy to follow, e.g., in a static analysis that is customized to handle the idioms. To inhibit analysis, we extended the mini-debugger with stealthier interfaces.

This interface consists of two aspects, as the exception-throwing process (1) needs to invoke the minidebugger in the exception-catching process by raising a signal, and (2) needs to pass the necessary information to identify the target code to be executed once control is transferred. Whereas our original design only allowed for a single implementation of both aspects, we explored and implemented several stealthier, alternative methods in our improved design for both of them

Moreover, the identification of target code fragments is also more flexible in the new design, as control can now be passed to any code fragment in the exception-catching process, whichever of the two processes is the exceptioncatching one at some point in time. In the original design by contrast, control could only be passed to the set of migrated code fragments. Because the identification of target code is more flexible, the interface through which both the mini-debuggers in both the processes are invoked can be exactly the same for invocations in both directions of the reciprocal design. This is a useful feature for two reasons. First, one mini-debugger implementation then suffices for both processes, so only one mini-debugger needs to be 
injected into the protected binary. Secondly, having the same interface in both directions implies that the static code fragments making invocations do not reveal which mini-debugger they invoke by means of their appearance alone. This reduces the amount of useful information that attackers can extract from the protected binary through simple static attacks such as pattern matching.

3.2.1. Signaling the Mini-Debugger. We replaced the conspicuous BKP T instructions of our original design with injected code that looks harmless and similar to the surrounding code, yet intentionally generates software faults. Whereas originally a SIGTRAP signal was generated and examined by the mini-debugger, our new design can handle a mix of different signals such as SIGSEGV and SIGFPE. Examples of injected code signaling the minidebugger from both the application and self-debugger processes can be observed in Figure 2 (a) and (g)). Here, ADR is an ARM assembler macro-instruction that produces an address in a register. The values $\mathrm{OBF} 1, \mathrm{OBF} 2$, and $\mathrm{OBF} 3$ produced by the ADR instructions are addresses that cause segmentation faults when accessed, either because they are invalid or because more permissions are required. We thus have some faulting instructions ( $\mathrm{LDR}, \mathrm{BX}$, and STR in Figure 2), preceded by some other instruction(s) that set up the fault (the ADR instructions in Figure 2).

Figure 2 depicts the injection of both the instructions that set the registers to set up the fault and the faulting instruction itself, such that they directly follow each other. Our implementation has more complex features to inhibit analyses. Instead of injecting faulting instructions, it can reuse instructions already present in the application. It then injects instructions that set up the registers for the software fault and add a jump to an already existing fragment that ends with an exception-causing instruction (e.g., a BX or a STR) when executed on the set register contents. This reuse makes the attacker's analyses harder as some instructions originally executed in only one context (the original application) are now executed in multiple contexts, i.e., both as parts of the original application code and to generate faults. It also splits the code that generates the software fault from the code that actually wants to invoke the mini-debugger, separating them in space. It even separates the software fault and its set-up in time when the jumped-to fragment contains instructions before the faulting instruction. Proper care then has to be taken that the intervening instructions do not change any live registers or have other side effects, of course.

By using less conspicuous code for signaling, however, it also becomes harder for the mini-debugger to distinguish between "forged" signals that request the switch to application code, and signals generated by genuine software faults. Causes of such signals can be bugs in the application, but also intentional functionality, such as processor feature detection, i.e., testing whether a certain instruction is supported by the hardware. Consequently, it is necessary for the mini-debugger to correctly make this distinction, and it thus contains a mechanism that allows for the verification of requested switches.

3.2.2. Target Identification. There are two aspects of target identification to consider: the location in which to pass the information about the target, and the way in which this information encodes the identity of the target. In our original design, both of these aspects were problematic with respect to stealthiness and ease of reverse engineering. First, the mapping table used for converting identifiers into addresses was a single centralized and unobfuscated table of all the addresses of migrated code fragments. A reverse engineer could thus, upon finding this table, find all control flow targets and start analyzing them. Secondly, in our original design the identifier was passed in a fixed place-the top of the stack-by the instruction immediately preceding the trap.

In our improved design, we take a decentralized approach: rather than passing an identifier that needs to be linked to a target somehow, we now pass the address of the requested target itself, in an obfuscated form. This way, there is no single table that can be abused by attackers to find all control flow targets. Moreover, the obfuscated address is no longer passed in a fixed location, but in a variable location that is implicated in the software fault used to signal the mini-debugger. This approach can be observed in Figure 2. In one instance, the code fragment in the self-debugger signals the mini-debugger by jumping to the address $\mathrm{OBF} 2$ (()), for which the execution memory permission is lacking. The mini-debugger finds this obfuscated address $\mathrm{OBF} 2$ by inspecting the software fault, and then decodes it into the target address to be invoked. Another example would be a a software fault caused by a division by zero, where the obfuscated address could be stored in the register that was used as the numerator.

In our current proof-of-concept implementation we deploy only a few methods to obfuscate the target addresses. This could easily be extended to using a myriad of data obfuscation methods. For every invocation the minidebugger would then employ a decision tree to determine the specific method to decode the obfuscated address. The variables used in the decision tree could include the signaling method, the values in fixed or method-dependent memory addresses or registers, etc.

\section{Implementation Challenges and Solutions}

This section discusses noteworthy aspects of our implementation that featured significant research challenges. In the process of exploring a more capable mini-debugger, we came to rely on more recent ptrace functionality such as PTRACE_SEIZE and PTRACE_O_EXITKILL [11]. These features are only present as of Linux 3.4 and 3.8, respectively. Our binary rewriter operates on ARMv7 binary code.

\subsection{Reciprocal Debugging without Deadlocks}

A system of two processes that debug each other and communicate via signals is fragile and prone to deadlocks. To make it work correctly took significant research efforts.

When a signal other than SIGKILL arrives at a debuggee process, the kernel places this process in a stopped state [11]. The debugger is notified of the signal, handles it (however it chooses to do so), and decides whether or not it is to be delivered to the debuggee. The debugger can thus choose to suppress signals, which in effect the minidebugger does when handling a request to transfer control to some application code. After the debugger is done 
handling the signal, it allows the debuggee to continue (with or without actual delivery of the signal). In essence, when a signal arrives at a debuggee process, it is stopped, and can only continue after its debugger allows it to. In the context of reciprocal debugging, this can become a problem. When signals arrive at both the protected application and the self-debugger simultaneously or close together, both processes end up in a stopped state, each waiting until the other one allows it to continue.

Conceptually, avoiding such deadlocks is simple: One can prevent signals arriving close together to both processes by preventing signals from arriving at the exception-catching process altogether. That process will then at all times be available to handle the signals arriving at the exception-throwing process. This is easier said than done, however. Avoiding deadlocks requires recognizing all possible sources of signals, and implementing methods to handle these cases. We describe several specific sources of signals, the associated deadlocks, and the solutions. We conclude by discussing the remaining possible deadlocks.

4.1.1. Child Processes. When something happens to a child process of potential interest to the parent processsuch as the child process stopping or exiting-, the kernel by default sends a SIGCHLD signal to that parent [21]. In addition, the kernel always treats a debugger process as a parent process of the debuggee [11], so whenever a debuggee changes state, its debugger is notified through a SIGCHLD signal. In our case, any state change in any of the two processes results in a SIGCHLD signal being sent to the other process. When the exception-throwing process invokes the active mini-debugger, this could cause a deadlock. Without precautions, the exception-throwing process is placed in a stopped state by the kernel, which simultaneously sends a SIGCHLD signal to the exception-catching process, which will be blocked until the exception-throwing process allows it to continue. This is precisely the sort of situation we need to avoid.

To do so, we let both the application and the selfdebugger process make use of the sigaction API [22] to disable the default sending of SIGCHLD signals by the kernel upon state changes in their child processes.

For applications where receiving and handling such signals from other child processes is part of the application's correct functioning, simply disabling the signals is not an option. An alternative solution, which we have not implemented yet but only requires some engineering, is then to keep these signals pending using the sigprocmask API [23], and only handle them at specified synchronization moments, when it is certain the self-debugger's process state will not change.

There is one exception to the ignoring of SIGCHLD signals: When a debugged process exits, the kernel always sends one, which cannot be disabled. However, before a debugged process is allowed to exit, its debugger is notified of the impending departure. The debugger can use this opportunity to detach from the exiting process, forestalling the arrival of the SIGCHLD signal. We hence solved this exception by making the self-debugger detach from the protected application in the appropriate places.

4.1.2. Users and IPC. A user or process can send any kind of signal directly at a process using the kill command or API, provided that they possess the required privileges [24]. Such signals might be used to request that the application output a status update, or in the context of inter-process communication (IPC). While we might question the motives of a human user simultaneously sending a signal to both the protected application and the self-debugger, there is at least one system process that has a good motive. When a system shutdown is requested, the system daemon-be it sysvinit, systemd, or something else-handles this by sending a SIGTERM signal to all running processes at the same time [25]. Most processes will then terminate, and any process that has not yet done so after a specified delay will be sent an additional SIGKILL, immediately terminating it. For reciprocal debugging, the initial SIGTERM causes a deadlock that not even the subsequent SIGKILL can solve.

Using the aforementioned sigaction API does not suffice now: When a debuggee process asks not to be notified of signals from a specific type, the kernel still informs its debugger [11], [22], which then first needs to intervene. In our case, we hence still have a deadlock. A working alternative is blocking the sending of signals of a certain type with the sigprocmask API [23]. Those signal are then kept pending until the type is unblocked again. For a debugged process, only when the signal is unblocked again will the debugger be notified of its arrival.

Whenever a mini-debugger becomes the active one, it therefore blocks all possible signal types except for SIGKILL and SIGSTOP, which we discuss later. This blocking is undone right before deactivating and transferring control to application code. The exceptioncatching process thus blocks all possible signals, while the exception-throwing process maintains its original behavior. This maintains behavior that depends on signals sent by other processes or users. The previously discussed case of a system shutdown serves as an example. There, the exception-throwing and the exception-catching processes simultaneously receive a SIGTERM signal. The signal arriving at the exception-catching process is simply blocked. The signal arriving at the exception-throwing process is first presented to the exception-catching process, which sends it on to the former. The application then shuts down in a proper manner, cleaning up properly before exiting.

4.1.3. Software Faults. The aforementioned solutions only block signals that were sent to it through kill or one of its variants. A signal caused by a software fault inside the process itself cannot be blocked that way, and has to be handled before the process can continue.

Faults inside the exception-throwing process can be intentional, i.e., injected by us to invoke the mini-debugger, or genuine faults, possibly caused by bugs or, e.g., by code testing the processor's support for certain instruction set extensions. In Section 4.3, we describe an optional mechanism to distinguish between these cases and to pass any genuine signal on to exception-throwing process. It is thus no problem if that process generates a genuine software fault. On the other hand, if the exception-catching process generates such a fault, a deadlock is certain to follow. A software fault generated in the exception-catching process can only be the result of a bug in the mini-debugger, so with proper engineering this becomes a non-issue. 
4.1.4. Remaining Deadlocks. An important caveat to the above is that neither SIGKILL nor SIGSTOP can be ignored or blocked, as described in Section 4.1.2. If a SIGKILL arrives at either process, it simply dies, taking the other process with it because of the PTRACE_O_EXITKILL option. This is not a problem, it is exactly what we want. If a SIGSTOP arrives at the exception-catching process, a deadlock does still occur, as the exception-throwing process is running application code instead of paying attention to its debuggee. This means that no SIGSTOP should ever be sent to the protected application or the self-debugger, as either could be the exception-catching process at any moment.

This is certainly a limitation, but is not necessarily an important one for the specific applications we want to protect. On the one hand, an application sending a SIGSTOP signal to stop itself or one of its component processes can be adapted to send a SIGTSTP signal instead. Contrary to a SIGSTOP, a SIGTSTP can be blocked. Although the resulting process stop might be delayed, no deadlock will occur. On the other hand, SIGSTOP signals originating outside of the application are entirely out of our control. Such signals are of use to potential attackers that want to to tamper with the application, however, and the resulting deadlock could hence be seen as extra protection.

\subsection{Signaling the Mini-Debugger}

We use intentional processor faults to signal the mini-debugger. Such faults can be generated in various ways, but ultimately result in just four different signals: SIGILL, SIGFPE, SIGSEGV, and SIGBUS. Popov et al. used such intentional faults for static control flow obfuscation [26]. Our context differs in two ways. First, whereas they aim to thwart disassembly, we aim to thwart hostile debuggers. Second, we use the ARMv7 architecture, which is used on a variety of platforms with many optional features. Portability is thus a major concern. We investigated the portability of various signaling methods for the ARMv7 architecture, and also compared with $\mathrm{x} 86$ (or x86-64) architectures to provide a broader scope.

4.2.1. Illegal Instructions. Not all ARM or $x 86$ processors implement all extensions of their respective instruction set architectures (ISAs). Moreover, in both ISAs many combinations of bits do not encode an actual instruction. For any processor, unsupported encodings are illegal instructions, and attempting to execute them results in a fault. On Linux, such a fault generates a SIGILL signal.

Inserting an illegal instruction encoding is not futureproof, however, as future ISA revisions could redefine such encodings. Unimplemented instructions differ between processors, and are hence not portable. Furthermore, instances are easy to identify using a disassembler.

4.2.2. Division by Zero. A less conspicuous approach uses instructions that only fault under some conditions, but otherwise execute normally. A good example are divisions, which only fault for division by zero. Using data obfuscation techniques, loading a zero into the denominator register can remain hidden from static analysis.

Divisions by zero are not portable, however. In languages like $\mathrm{C}$, they are undefined and hence their behavior can vary from one system to another. While on Linux x86 systems, an attempted division by zero generates a SIGFPE signal, this is not necessarily the case for ARM Linux. Most ARM processors do not implement the UDIV and SDIV instructions intended for integer division, emulating these in software instead. On such processors, trying to divide by zero with the UDIV instruction would not result in a SIGFPE, but rather in a SIGILL, as the instruction is not even recognized. On other ARM systems (or architectures such as MIPS), no fault or signal is even generated, and the division by zero simply returns a zero.

4.2.3. Segmentation Faults. A segmentation fault occurs upon an invalid memory access. The access might be invalid because the address does not refer to mapped memory at all, such as a NULL pointer dereference; or because the process lacks the permissions required for the specific access, such as writing to read-only memory, or trying to execute kernel code. On Linux, a segmentation fault results in a SIGSEGV signal.

We can intentionally generate segmentation faults by inserting instructions that makes invalid memory accesses. The layout of the address space is not very predictable, however. It contains two fixed areas that we can choose addresses from: the NULL page, and kernel space. Even if the kernel is running with Kernel Page Table Isolation (to protect against Meltdown attacks), accessing locations in the then unmapped kernel memory still results in a SIGSEGV. The kernel's exact address range varies between architectures, and some pages are explicitly mapped to be accessible from user space such as the vectors page on ARMv7 and the vsyscall page on x86. As their addresses are fixed, they pose no real problems.

On ARM, we have two choices for the specific faulting instructions: load/store instructions and indirect controlflow instructions. At the moment of the attempted memory access or control transfer, their base register and target address register contain the kernel-space address, which can be computed in an obfuscated manner to make it less conspicuous. Many more options to generate segmentation faults exist, and the options for which the specific fault address can easily encode information include the following: writing to the binary's read-only data segment, branching to the binary's data segment, branching to the stack, etc. On x86, equivalent instructions exist that can be used to trigger segmentation faults.

4.2.4. Unaligned Memory Accesses. Even if an instruction accesses a valid address, it can still fault when the address is not alignment. Some ARM processors do not allow any unaligned memory access. Some only disallow it for certain instructions. Similarly, some x 86 instructions also require a specific alignment. In those cases, unaligned memory accesses are regarded as a software fault, and on Linux result in a SIGBUS signal.

As the instructions that can trigger a SIGBUS vary across ARM processors, there is a clear portability issue. We can sidestep this issue by only inserting instructions for which unaligned memory accesses are illegal on every ARM processor, such as LDREX, but that is not stealthy at all. Moreover, a more problematic portability issue is that the Linux kernel on ARM is often configured to hide unaligned memory accesses from user processes. In 
TABLE 1. OVERHEAD OF INDIVIDUAL SELF-DEBUGGING ASPECTS

\begin{tabular}{|l|c|}
\hline Aspect & Execution Time \\
\hline Initialization & $16 \mathrm{~ms}$ \\
Memory Read & $4.4 \mu \mathrm{s}$ \\
Memory Write & $4.8 \mu \mathrm{s}$ \\
Switch SIGTRAP & $7.9 \mathrm{~ms}$ \\
Switch SIGSEGV (RW) & $0.065 \mathrm{~ms}$ \\
Switch SIGSEGV (X) & $0.060 \mathrm{~ms}$ \\
\hline
\end{tabular}

that case, the kernel handles unaligned memory accesses without even sending a SIGBUS signal.

4.2.5. Actual Implementation. From our investigation we concluded that triggering segmentation faults is the most platform-independent signaling method. It can be made to work on all ARM devices, and it is also portable to $\mathrm{x} 86$ platforms. In general, we suspect significantly less portability concerns on the $\mathrm{x} 86$ platform. The two signaling methods we implemented in our prototype reflect our conclusions. Both methods use segmentation faults: one uses loads and stores, the other uses indirect branches.

\subsection{Switch Verification}

Upon invocation, the mini-debugger has to determine whether it is dealing with an actual invocation or a genuine signal caused by original application behavior. We therefore use an allowlist that contains the addresses of all potential faulting instructions, rather than a full bidirectional mapping. If the fault address is not allowed, the signal is genuine and the mini-debugger passes it on to the exception-throwing process. The addresses in the allowlist are, like those in the original mapping table, of considerable interest to attackers. They should therefore be stored in a hashed manner, similar to passwords.

\section{Evaluation}

We evaluated and tested our technique on an Arndale ARMv7 Board that runs Ubuntu 15.04, featuring Linux 4.3.0. In our tool chain, we used GCC 4.8.1 and GNU binutils 2.23 , the latest versions for which we have the necessary patches to support our binary rewriter. From version 4.4 onwards, Android features Linux kernels recent enough for our improved mini-debugger, but we lack a patched Android compiler tool chain for such recent versions. We therefore did not evaluate our technique on Android. The lack of recent patches is an engineering issue that results from a lack of resources to port and maintain patches forward, not a fundamental issue. All patches are available online, and all our prototype source code will be released upon publication of this paper.

\subsection{Performance Overhead}

It is rather meaningless to present a concrete performance overhead for an application protected by our improved self-debugging technique. When protecting their application, developers choose which code fragments to migrate to the self-debugger in function of the specific, sensitive code they want to protect. To thwart attackers, they should ensure plenty of control flow switches occur
TABLE 2. RESULTS FROM BINARY NINJA ANALYSIS

\begin{tabular}{|c|cc|cc|cc|}
\hline $\begin{array}{c}\text { \# Code } \\
\text { Fragments }\end{array}$ & \multicolumn{2}{|c|}{ Migrated Targets } & \multicolumn{2}{c|}{ Non-migrated Targets } & \multicolumn{2}{c|}{ Faulting Instructions } \\
Disassembled & Total & Disassembled & Total & Value Found & Total \\
\hline 3 & 2 & 3 & 1 & 5 & 3 & 9 \\
5 & 2 & 5 & 4 & 7 & 5 & 14 \\
7 & 2 & 7 & 6 & 9 & 4 & 19 \\
10 & 2 & 10 & 4 & 12 & 7 & 25 \\
13 & 3 & 13 & 8 & 15 & 6 & 32 \\
15 & 4 & 15 & 10 & 17 & 10 & 36 \\
\hline
\end{tabular}

in or near the sensitive code. The overhead for a specific application depends by and large on the fragments being migrated and on which code paths these fragments lie. Profiling can be used to select code fragments that are not too hot, nor too cold, but the exact performance overhead is context-dependent. On top of that, we can transform hot code to create colder code that is more suitable to being migrated, through loop tiling for example.

Still, we can measure the impact of several operations, such as its initialization, memory operations performed from a migrated code fragment, and switches to and from such a fragment. To that extent, we used microbenchmarks that were completely dominated by the aspect to measure, compiled these benchmarks at -02 and employed perf. Our results can be seen in Table 1. We measured the execution time for: the self-debugging initialization routine; memory reads or writes from the self-debugger, using the mini-debugger's memory support; and control flow switches, using the different signaling methods we implemented. The memory reads and writes correspond to a single LDR or STR instruction. Two switches occur when executing a migrated code fragment in the self-debugger: one switch to the self-debugger, and one switch back the application.

We measured the SIGTRAP signaling method employed in our previous implementation as a reference, and came to the conclusion that it is two orders of magnitude slower as the stealthier signaling methods we implemented. The reason for this is that the specific Linux kernel code path triggered by a BKP T instruction performs some I/O, slowing it down [27].

\subsection{Security Evaluation}

We evaluate how our improved design thwarts several approaches an attacker could take to defeat our original design, and conclude this section by considering the attack approaches that remain, as well as the orthogonal defenses that can improve the resilience of our technique.

In our original design, an attacker could attach their own debugger to the self-debugger. In our improved design, such a debugger can no longer attach to either the self-debugger or the protected application. We verified this in real life, but also investigated an alternative attack that, with some extra work, still allows an attacker to attach a hostile debugger to either process, although only temporarily. We explain this attack with the example goal of attaching to the protected application. To achieve this goal, the attacker needs to simultaneously terminate the selfdebugger through a SIGKILL, and pause the protected application through a SIGSTOP. The latter signal is necessary to prevent the protected application from continuing its execution, encountering a mini-debugger invocation masquerading as a software fault, and dying. The attacker 
can subsequently attach their debugger to the application, continue it, and analyze or tamper with its execution until it encounters the next software fault. To perform this attack the PTRACE_O_EXITKILL option first has to be disabled, however. If it were not, the SIGKILL signal would result in the death of both processes.

After tampering with the binary to disable PTRACE_O_EXITKILL, an attacker can thus temporarily attach to either process and observe or tamper with the execution of application code (migrated or otherwise). To stay attached more permanently, however, the attacker has to reverse the transformations our technique employs to migrate code and invoke the mini-debugger. In our previous design, such invocations could easily be recognized statically through their use of $B K P T$ instructions. In our improved design, this is no longer the case as we reuse application code to trigger software faults. If an attacker were to invest extra time and effort, a more advanced static analysis might be able to find the faulting instructions, however.

To evaluate how easy it was to find faulting instructions statically, we set up an experiment using the opensource compression tool bzip2, compiled at -Os. We successively migrated more code fragments to the selfdebugger, and analyzed the resulting binary using Binary Ninja [28]. The reason we use Binary Ninja as opposed to other disassemblers (such as IDA Pro) is that it contains more advanced-and for this experiment, more relevantfeatures such as value-set analysis (VSA). Table 2 contains the results of Binary Ninja's analysis. The only protection we applied to bzip2 was our own self-debugging technique; we did not use any obfuscations. Even then, VSA found the faulting address as a possible value for only roughly a third of all faulting instructions. Binary Ninja does not explicitly flag these values as causing software faults, but an attacker could write an analysis to do so. On top of that, Binary Ninja's recursive descent no longer disassembles all the targets of control transfers, regarding them as data instead. The control transfers from the mini-debugger to these targets happen through a complex indirection, and can not be followed. Some targets have more direct incoming control flow that causes them to be disassembled, however, or are recognized as code without incoming control flow by heuristics. Our stealthier interfaces are thus rather effective in hiding mini-debugger invocations, but could be even more effective if augmented with more advanced data obfuscations.

If attackers cannot locate and reverse our transformations statically, they have to do so dynamically. Our technique's entire purpose is to hinder dynamic analysis using debuggers, however. Reversing our transformations one software fault at a time would come at a considerable cost. Our technique can be made even stronger by applying orthogonal defenses such as (data) obfuscations and integrity checks, particularly on the initialization routine where the PTRACE_O_EXITKILL option is enabled. Other, less convenient, potential approaches still left open to an attack are emulation, which we consider out of scope; and some sort of emulation/interposing of the ptrace API, which would again require considerable effort.

\section{Conclusions}

We improved the state-of-the-art anti-debugging technique of self-debugging by introducing reciprocal debugging and by making the interfaces to the self-debugger stealthier. The goal of these improvements was to make it harder for attackers to defeat the protection. Our evaluation confirms that this goal was reached.

\section{References}

[1] J. Nagra and C. Collberg, Surreptitious Software: Obfuscation, Watermarking, and Tamperproofing for Software Protection, 1st ed. Addison-Wesley Professional, 2009.

[2] B. De Sutter, P. Falcarin et al., "Preliminary ASPIRE Security Model," ASPIRE Consortium, Deliverable D4.01 v1.0, 2014.

[3] B. Wyseur, B. De Sutter et al., "ASPIRE Attack Model," ASPIRE Consortium, Deliverable D1.02 v2.1, 2016.

[4] P. Ferrie, "The Ultimate Anti-Debugging Reference," 2011. [Online]. Available: http://anti-reversing.com/Downloads/ Anti-Reversing/The_Ultimate_Anti-Reversing_Reference.pdf

[5] H. Miller, "Beginners Guide to Basic Linux Anti-Anti-Debugging Techniques," CodeBreakers Journal, 2005.

[6] Carbon Monoxide, "ScyllaHide," 2019. [Online]. Available: https://github.com/x64dbg/ScyllaHide

[7] Mrexodia, "TitanHide," 2019. [Online]. Available: https://github. com/mrexodia/TitanHide

[8] Ferrit, "OllyExt 1.8," 2014. [Online]. Available: https://tuts4you. com/download.php?view.3392

[9] Pellsson, "Starcraft 2 Anti-Debugging," 2010. [Online]. Available: https://tinyurl.com/tyxjkeb

[10] P. Ferrie, "Anti-Unpacker Tricks," in CARO, 2008.

[11] Linux Programmer's Manual, "ptrace(2)." [Online]. Available: http://man7.org/linux/man-pages/man2/ptrace.2.html

[12] BSD System Calls Manual, "PTRACE(2)."

[13] M. Russinovich et al., Windows Internals. Microsoft Press, 2012.

[14] B. Abrath, B. Coppens et al., "Tightly-Coupled Self-Debugging Software Protection," in SSPREW. ACM, 2016, p. 7.

[15] M. Ceccato et al., "ASPIRE Security Evaluation Methodology," ASPIRE Consortium, Deliverable D4.06, 2016.

[16] _ - "Understanding the behaviour of hackers while performing attack tasks in a professional setting and in a public challenge," Empirical Software Engineering, vol. 24, no. 1, pp. 240-286, 2019.

[17] Linux Programmer's Manual, “fork(2)." [Online]. Available: http://man7.org/linux/man-pages/man2/fork.2.html

[18] N. Nethercote and J. Seward, "Valgrind: A Framework for Heavyweight Dynamic Binary Instrumentation," in PLDI, 2007.

[19] F. Bellard, "QEMU, a Fast and Portable Dynamic Translator." in USENIX ATC, 2005, pp. 41-46.

[20] R. Paleari et al., "A fistful of red-pills: How to automatically generate procedures to detect cpu emulators," in WOOT, vol. 41, 2009 , p. 86.

[21] Linux Programmer's Manual, “wait(2)." [Online]. Available: http://man7.org/linux/man-pages/man2/wait.2.html

[22] _ , "sigaction(2)." [Online]. Available: http://man7.org/linux/ man-pages/man2/sigaction.2.html

[23] —, "sigprocmask(2)." [Online]. Available: http://man7.org/ linux/man-pages/man2/sigprocmask.2.html

[24] —, "kill(2)." [Online]. Available: http://man7.org/linux/ man-pages/man2/kill.2.html

[25] Linux Standard Base Core Specification, Generic Part, "shutdown." [Online]. Available: https://refspecs.linuxbase.org/ LSB_5.0.0/LSB-Core-generic/LSB-Core-generic/shutdown.html

[26] I. V. Popov, S. K. Debray, and G. R. Andrews, "Binary Obfuscation Using Signals," in USENIX Security, 2007, pp. 275-290.

[27] J. Whitham, "The mystery of the fifteen-millisecond breakpoint instruction," 2015. [Online]. Available: https://tinyurl.com/wu76t3j

[28] Vector 35, "Binary Ninja." [Online]. Available: https://binary.ninja/ 